\title{
Electropolymerization of Indole and Its 5- Position-Substituted Derivatives in the Mixed Electrolytes of Acetic Acid and Boron Trifluoride Diethyl Etherate
}

\author{
Congcong Liu ${ }^{1,2, *}$, Peng Liu', Wenjun Ding ${ }^{2}$, Shuai $\mathrm{Zhu}^{2}$, Xia $\mathrm{Li}^{2}$, Jingkun $\mathrm{Xu}^{2, *}$ \\ ${ }^{1}$ Interdisciplinary Materials Research Center, Key Laboratory of Advanced Civil Engineering \\ Materials (Ministry of Education), School of Materials Science and Engineering, Tongji University, \\ Shanghai 201804, China. \\ 2 Jiangxi Key Laboratory of Organic Chemistry, Jiangxi Science and Technology Normal University, \\ Nanchang, Jiangxi 330013, China \\ *E-mail: 1cc.0705@163.com; xujingkun@tsinghua.org.cn
}

doi: $10.20964 / 2019.06 .57$

Received: 5 February 2019 / Accepted: 9 April 2019 / Published: 10 May 2019

The mixed electrolyte of acetic acid and boron trifluoride ethyl ether (BFEE), containing poly(ethylene glycol) with a molar mass of 1000 (PEG1000), was employed for the electrochemical synthesis of indole and its 5-position-substituted derivatives, such as 5-cyanoindole, 5-nitroindole, indole-5carboxylic acid, and 5-hydroxyindole. The introduction of acetic acid into BFEE increased both the solubility of PEG1000 and the ionic conductivity of the well mixed electrolyte. In this medium, highquality polyindole (PIN) and its derivatives were successfully electrodeposited via anodic oxidation of their corresponding monomers. The as-formed PINs exhibited good electrochemical behavior and good fluorescence properties.

Keywords: Mixed electrolyte, Polyindoles, Electrochemical polymerization, Boron trifluoride ethyl ether, Fluorescence

\section{$\underline{\text { FULL TEXT }}$}

(C) 2019 The Authors. Published by ESG (www.electrochemsci.org). This article is an open access article distributed under the terms and conditions of the Creative Commons Attribution license (http://creativecommons.org/licenses/by/4.0/). 